\section{Observations of Variability in Young Stellar Objects and Implications for Protoplanetary Carbon Reservoirs}

\author{
RACHEL L SMITH ${ }^{1}$, ADWIN BOOGERT ${ }^{2}$, GEOFF BLAKE ${ }^{3}$ \\ AND KLAUS M PONTOPPIDAN ${ }^{4}$ \\ ${ }^{1} \mathrm{NC}$ Museum of Natural Sciences/Appalachian State University \\ ${ }^{2}$ University of Hawaii \\ ${ }^{3}$ California Institute of Technology \\ ${ }^{4}$ Space Telescope Science Institute \\ Presenting Author: rachel.smith@naturalsciences.org
}

High-resolution astronomical observations of $\mathrm{CO}$ toward young stellar objects (YSOs) provide a window into protoplanetary systems with relevance to solar system analogues and the early solar nebula. As a major reservoir of carbon and a key precursor molecule for $\mathrm{H}_{2} \mathrm{CO}$ and $\mathrm{HCN}$, and $\mathrm{CO}_{2}, \mathrm{CO}$ is a fundamental component in early prebiotic chemistry in evolving planetary systems, yet a comprehensive understanding of these chemical pathways is lacking. Previous observational studies of YSOs have provided insights into protoplanetary phenomena including $\mathrm{CO}$ self-shielding, supernova inheritance in the nebular cloud, and the interplay between $\mathrm{CO}$ ice-gas reservoirs. Toward furthering our understanding of the short-term evolution of carbon-based reservoirs in a range of YSO environments, we have begun an observational study exploring variability in $\mathrm{CO}$ gas-phase abundances and temperatures over several epochs for a group of massive YSOs from near our local solar neighborhood to the Galactic center. CO rovibrational absorption spectra were obtained using NASA's IRTF observatory with the iSHELL instrument at very high spectral resolution $(\mathrm{R} \sim 80,000)$ in the $M$ bands ( $v=1-0)$ for optically thin ${ }^{13} \mathrm{C}^{16} \mathrm{O},{ }^{12} \mathrm{C}^{18} \mathrm{O}$, and ${ }^{12} \mathrm{C}^{17} \mathrm{O}$, and $K$-bands $(v=2-0)$ for ${ }^{12} \mathrm{C}^{16} \mathrm{O}$. Molecular column densities for each YSO were derived by fitting each spectral line with a Gaussian and measuring optical depths using the mean line width from ${ }^{12} \mathrm{C}^{18} \mathrm{O}$ lines. Initial findings from our massive YSOs as well as from low-mass YSO data from preliminary work (example spectra for solar analogue HL Tau observed with VLT-CRIRES, Fig. 1), suggest that some $\mathrm{CO}$ reservoirs, in particular ${ }^{13} \mathrm{C}^{16} \mathrm{O}$ and its ratios with respect to ${ }^{12} \mathrm{C}^{16} \mathrm{O}$ and ${ }^{12} \mathrm{C}^{18} \mathrm{O}$, vary significantly in abundance over short timescales (Fig. 2). Gas temperatures show significantly less variation $(\leq 13 \%)$, and column density variation for the other isotopologues is less apparent. These findings thus far suggest that differences in abundance variation could signify disparate isotopologue-dependent chemical pathways for key carbon molecules. Further, such variability could be a signature of inheritance of isotopic heterogeneity from reservoirs in the parent cloud to the planetary nebula. This ongoing observational study using IRTF-iSHELL will add statistical significance to these initial findings, which could also be further explored in interdisciplinary work with solar system materials.
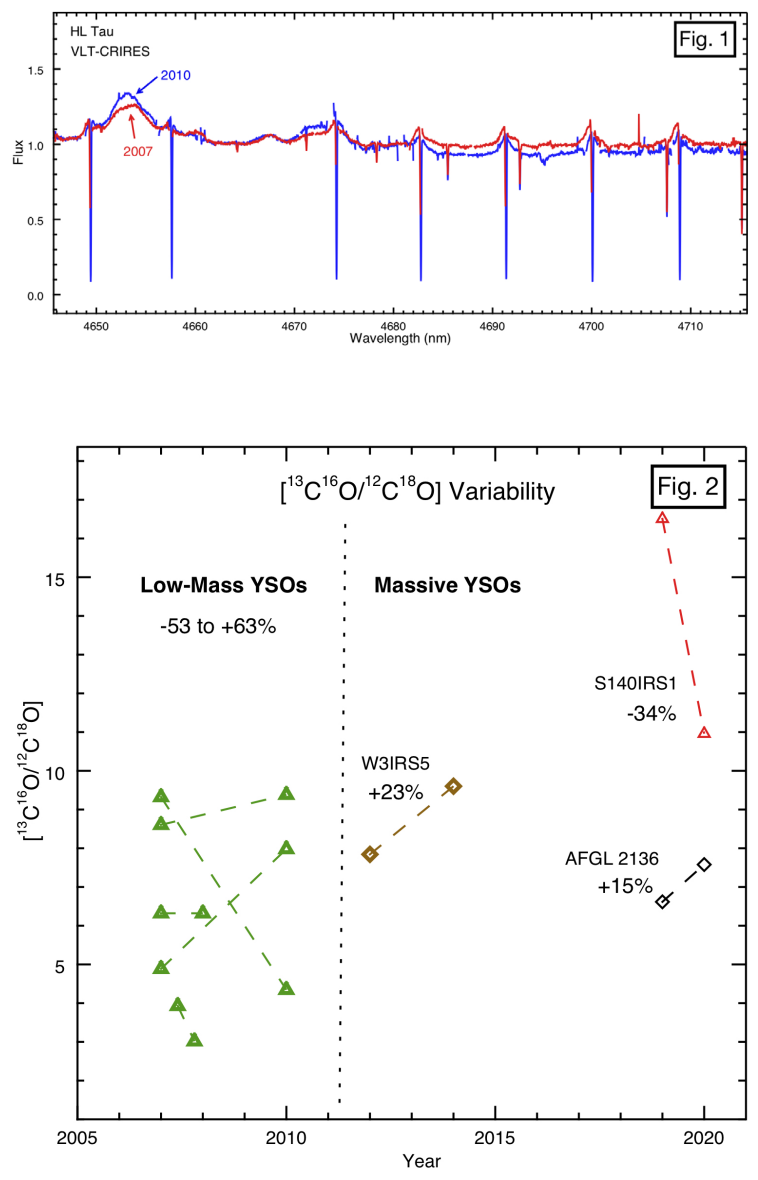